\title{
Semblanza científica del Prof. F.D. Calonge con motivo de su jubilación
}

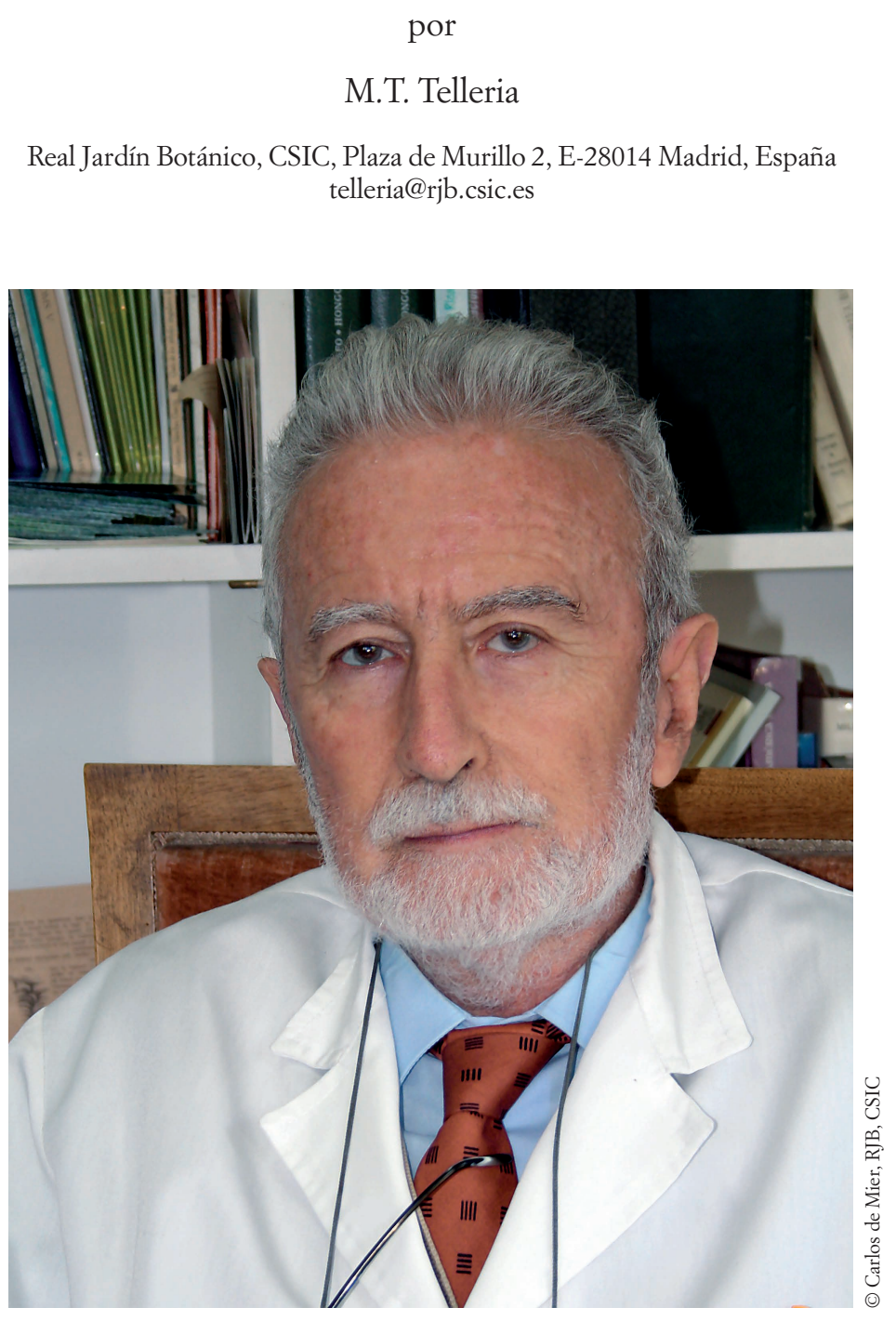

El momento de la jubilación es inexorable, a plazo fijo, y a Francisco de Diego Calonge le llegó el 28 de enero de 2008. El Prof. Calonge ha dedicado su actividad científica al estudio de los hongos, compaginando su dilatada trayectoria investigadora con la de divulgador, lo que le ha convertido en una de las figuras clave de la micología española de los últimos cuarenta años.
Su carrera científica comenzó a principios de los sesenta cuando, tras licenciarse en Farmacia por la Universidad Complutense de Madrid, realizó su tesis doctoral en el campo de la fitopatología. Al acabar ésta, en 1965, se trasladó a la Universidad de Bristol (UK) para continuar con el estudio de los hongos fitopatógenos y más concretamente con el análisis ultra- 
estructural de los hongos parásitos, campo en el que realizó importantes aportaciones al conocimiento de las relaciones parásito/hospedante. Recibió una sólida formación científica, adelantada a su tiempo, atípica para la época en nuestro país al menos en el ámbito de la botánica.

A su regreso a España, en 1968, se incorporó a la plantilla del Real Jardín Botánico (CSIC), donde ha desarrollado toda su labor profesional. Obtuvo por oposición la plaza de Colaborador Científico del CSIC (1972), después la de Investigador (1975) y, en 1982, la de Profesor de Investigación. Dirigió el RJB entre 1979 y 1984.

Su reincorporación al sistema científico español de los años sesenta no fue fácil, pues la penuria de medios y la incomprensión del momento le impidieron seguir con sus estudios fitopatológicos, y tuvo que iniciar una nueva etapa, con una línea de investigación que pudiera implementarse con menos recursos. Comenzó así con el estudio florístico y taxonómico de los macromicetos. Ascomycetes, Agaricales, Aphyllophorales y, sobre todo, Gasteromycetes, en los que es un especialista a nivel mundial, han sido los grupos en los que ha centrado, desde entonces, su atención. Ha dedicado la mayor parte de su esfuerzo científico a la elaboración de catálogos florísticos y floras, monografías, aportaciones corológicas y descripción de especies nuevas. Comenzó sus estudios en el ámbito geográfico español, para después ampliarlo al europeo: Polonia, Italia, Ucrania y, sobre todo, Portugal; país éste en el que últimamente se ha centrado en la biota fúngica de Madeira. El salto al continente americano se produjo a mediados de los noventa, con el inicio de la publicación de una serie de contribuciones al conocimiento de los hongos de Venezuela, Bolivia, Brasil, Ecuador, Guatemala y, sobre todo, México y Costa Rica. Tanzania y Camerún en África tropical e India en Asia completan la lista de países cuya biota fúngica ha merecido su atención.

El Prof. Calonge ha sido un precursor de la divulgación científica en España y uno de sus máximos exponentes en el campo de la micología. Fundó la Sociedad Micológica de Madrid y ha sido su "alma mater", además de Presidente, durante una parte muy importante de su larga singladura. Ha organizado las exposiciones anuales de hongos que, cada otoño, desde 1973 se han realizado en Madrid. Fundó el Boletín de la Sociedad Micológica de Madrid-Boletín de la Sociedad Micológica Castellana hasta su n. ${ }^{\circ}$ 10, de diciembre de 1985-y lo dirigió desde sus comienzos, en 1976, hasta 2008. Conferencias, cursos, programas de televisión y radio, artículos de prensa y un buen número de libros, jalonan este apartado de su currícu- lum. De entre los libros destacaré, por ser el primero que publicó, en 1975, "Hongos de nuestros campos y bosques", del que se hicieron hasta tres ediciones el mismo año. Fue este manual un punto de referencia obligado para muchos aficionados que andaban perdidos en el panorama editorial español de aquellos años, escaso en publicaciones dirigidas a los micólogos amateurs.

Pero esta vocación pedagógica no quedó limitada al campo de la divulgación ya que F.D. Calonge se volcó, también, en la formación de jóvenes investigadores mediante la dirección de tesis doctorales; once en total. Esta labor fue el germen de una nueva generación de micólogos que ha contribuido a restablecer y consolidar la micología en nuestro país. Se recuperó así la escuela micológica del Real Jardín Botánico que Romualdo González Fragoso fundara en los primeros años del siglo XX y que declinó, a principios de los años sesenta, con la muerte de Jordán de Urríes.

El 2 de julio de 1979, F.D. Calonge es nombrado Director del Real Jardín Botánico y, desde esa fecha, hasta principios de diciembre de 1984 en que deja el cargo, realizó una importante labor al frente del mismo. Tras una ejemplar restauración, reabrió el Jardín al público (1981) y, bajo su dirección, se inició una de las etapas más brillantes y prolongadas de la Institución.

Muchos méritos más, científicos y profesionales, jalonan su currículum vítae, pero son en mi opinión sus valores personales los que más destacan en su dilatada carrera. Su infatigable entrega al trabajo y su vocación investigadora destacan sobre todos ellos. Por eso no es de extrañar que, en este caso, la jubilación haya sido tan sólo un trámite administrativo que no ha retirado al Prof. Calonge de seguir dedicándose, por entero, a su pasión científica: la micología.

\section{RELACIÓN CRONOLÓGICA DE SUS PUBLICACIONES}

\section{5}

Rubio-Huertos, M. \& Diego [Calonge], F. de. 1965. Estudio histo-citológico, mediante microscopía normal y electrónica, de la infección por Puccinia hordei Otth. Microbiología Española 18: 127-142.

\section{7}

Calonge, F.D. 1967. Chlorophyll and total nitrogren in barley rust infection. Transations of the British Mycological Society 50: 397-401.

\section{8}

Calonge, F.D. 1968. Origin and development of intrahyphal hyphae in Sclerotinia fructigena. Mycologia 60: 932-942. 
[Diego] Calonge, F. de \& Rubio-Huertos, M. 1968. Características micro y ultramicroscópicas del parasitismo por Erysiphe graminis var. hordei. Anales del Instituto Botánico Antonio José Cavanilles 23: 211-234 [1965].

\section{9}

Byrde, R.J.W., Fielding, A.H. \& Calonge, F.D. 1969. The role of Pectolytic Enzymes of Sclerotinia fructigena in Fruit Rot. Phytochemistry 8: 1-2.

Calonge, F.D. 1969. The Occurrence of Glycogenmembrane Complexes in Fungi. An Electron Microscope Study. Protoplasma 67: 79-85.

Calonge, F.D. 1969. Electron Microscope Studies on Tomentella. I. Ultrastructure of the Vegetative Hyphae. Archives of Mikrobiology 65: 136-145.

Calonge, F.D. 1969. Ultrastructure of the Haustoria or Intracellular Hyphae in Four Different Fungi. Archives of Mikrobiology 67: 209-225.

Calonge, F.D. 1969. Ultrastructure of the hyphae of Phytophthora palmivora with special reference to intrahyphal hyphae and vesicular elements. Microbiologia Española 22: 97-111.

Calonge, F.D., Fielding, A.H. \& Byrde, R.J.W. 1969. Multivesicular Bodies in Sclerotinia fructigena and their Possible Relation to Extracellular Enzyme Secretion. Journal of General Microbiology 55: 177 184.

Calonge, F.D., Fielding, A.H., Byrde, R.J.W. \& Akinrefon, O.A. 1969. Changes in Ultrastructure following Fungal Invasion and the Possible Relevance of Extracellular Enzymes. Journal of Experimental Botany 20: 350-357.

Calonge, F.D. \& Torre, M. de la. 1969. Ultraestructura del parasitismo de Exobasidium sobre Rhododendron. Anales del Instituto Botánico Antonio José Cavanilles 25: 263-276 [1967].

Willetts, H. J. \& Calonge, F.D. 1969. The Ultrastructure of the Stroma of the Brown Rot Fungi. Archives of Mikrobiology 64: 279-288.

Willetts, H.J. \& Calonge, F.D. 1969. Spore Development in the Brown Rot Fungi (Sclerotinia spp.). New Phytologist 68: 123-131.

\section{0}

Calonge, F.D. 1970. Notes on the Ultrastructure of the Microconidium and Stroma in Sclerotinia sclerotiorum. Archives of Mikrobiology 71: 191-195.

Calonge, F.D. 1970. Estructuras cristalinas intracelulares en Sclerotinia fructigena. Microbiología Española 22: 199-207.

Calonge, F.D. 1970. Electron Microscope Studies on Tomentella II. Hyphal Anastomosis and Reproductive Organs. Mycopathologia et Mycologia Applicata 41:363-371.
Calonge, F.D. 1970. Determinación cualitativa de aminoácidos y azúcares libres en hoja de cebada sana e infectada con la roya. Microbiología Española 23: 195- 201.

Calonge, F.D. 1970. Estudios sobre hongos I. Algunos ejemplares colectados en Madrid y sus alrededores. Anales del Instituto Botánico Antonio José Cavanilles 26: 15-35 [1968].

\section{1}

Calonge, F.D. 1971. Estudios sobre hongos II. Contribución al catálogo de las provincias de Madrid y Segovia. Anales del Instituto Botánico Antonio José Cavanilles 27: 7-27 [1969-1970].

[Diego] Calonge, F.D. de. 1971. Hongos fitopatógenos de interés económico en el Jardín Botánico de Madrid. Acofar 62: 19-25.

\section{2}

Calonge, F.D. 1972. Estudios sobre hongos III. Aportación al catálogo de las provincias de Madrid y Segovia. Anales del Instituto Botánico Antonio José Cavanilles 28: 5-33 [1971].

Calonge, F.D. 1972. I Congreso Internacional de Micología. Anales del Instituto Botánico Antonio José Cavanilles 29: 144-145.

Calonge, F.D. 1972. III Reunión de Botánica Criptogámica. Anales del Instituto Botánico Antonio José Cavanilles 29: 145-146.

Calonge, F.D. 1972. I Cursillo de Micología. Anales del Instituto Botánico Antonio José Cavanilles 29: 146.

Calonge, F.D. 1972. La colección de Poliporáceos de Lázaro e Ibiza. Anales del Instituto Botánico Antonio José Cavanilles 29: 146-147.

[Diego] Calonge, F. de \& Torre, M. de la. 1972. Comentarios sobre algunas setas comestibles o venenosas que hay que conocer y diferenciar. Acofar 76: 10-19.

\section{3}

Calonge, F.D. 1973. Estudios sobre hongos IV. Aportación al catálogo de las provincias de Madrid y Segovia. Anales del Instituto Botánico Antonio José Cavanilles 30: 19-32.

Calonge, F.D. 1973. I Simposio de Botánica Criptogámica. Anales del Instituto Botánico Antonio José Cavanilles 30: 273.

Calonge, F.D. 1973. II Cursillo de Micología. Anales del Instituto Botánico Antonio José Cavanilles 30: 273-274.

Calonge, F.D. 1973. I Exposición de Hongos de Madrid. Anales del Instituto Botánico Antonio José Cavanilles 30: 274. 
Calonge, F.D. \& Zugaza, A. 1973. Catálogo de los hongos presentados en la I Exposición de Madrid del 1 al 5 de Noviembre de 1972. Anales del Instituto Botánico Antonio José Cavanilles 30: 33-55.

[Diego] Calonge, F. de, Torre, M. de la, Moreno, G. \& Vallejo, J.L. 1973. Algunos hongos interesantes de la Casa de Campo de Madrid. Acofar 87: 18-27.

Wright, J.E. \& Calonge, F.D. 1973. The location of Lázaro e Ibiza's collections of Polyporaceae. Taxon 22: $267-270$

\section{4}

Calonge, F.D. 1974. Hongos de Tenerife colectados durante la III Reunión de Botánica Criptogámica. Anales del Instituto Botánico Antonio José Cavanilles 31(1): 19-26.

Calonge, F.D. \& Moreno, G. 1974. Contribución al estudio micológico de la Sierra de Guadarrama. I. Novedades de Basidiomicetos para la flora española. Boletín de la Estación Central de Ecología 3(5): 23-28.

[Diego] Calonge, F. de. 1974. Las setas, un mundo enigmático y apasionante. Laboratorios Glaxo. Madrid. López-Pérez, M.J., Giménez-Solves, A., Calonge, F.D. \& Santos-Ruiz, A. 1974. Evidence of Glyoxysomes in Germinating Pine Seeds. Plant Science Letters 2: 377-386.

Torre, M. de la \& Calonge, F.D. 1974. Melastiza Boud. (Ascomycetes), género nuevo para España. Lagascalia 4: 43-47.

\section{5}

Bronchart, R., Calonge, F.D. \& Demoulin, V. 1975. Nouvelle contribution a l'étude de l'ultrastructure de la paroi sporale des Gastéromycètes. Bulletin de la Société Mycologique de France 91: 231-246.

Calonge, F.D. 1975. Aspectos micro y ultramicroscópicos de la Puccinia malvacearum Mont. Anales del Instituto Botánico Antonio José Cavanilles 31(2): 155-170 [1974].

Calonge, F.D. 1975. Ornamentación de las esporas de algunos Gasteromycetes españoles. Anales del Instituto Botánico Antonio José Cavanilles 32(2): 103. 115.

Calonge, F.D. 1975. Hongos de nuestros campos y bosques. ICONA. Madrid.

Calonge, F.D. 1975. Hongos de nuestros campos y bosques. 2. ${ }^{a}$ ed. ICONA. Madrid.

Calonge, F.D. 1975. Hongos de nuestros campos y bosques. 3. ${ }^{a}$ ed. ICONA. Madrid.

Calonge, F.D. \& Demoulin, V. 1975. Les Gastéromycètes d'Espagne. Bulletin de la Société Mycologique de France 91: 247-292.

[Diego] Calonge, F. de, Moreno-Horcajada, G. \& Telleria Jorge, M.T. 1975. Consideraciones sobre algu- nos hongos interesantes en bromatología y toxicología. Acofar 109: 10-19.

Moreno, G. \& Calonge, F.D. 1975. Contribución al estudio micológico de la Sierra de Guadarrama II. Algunos Basidiomicetos nuevos o raros para la flora española. Lagascalia 5: 3-14.

Moreno, G., Calonge, F.D. \& Torre, M. de la. 1975. Estudio ecológico y descriptivo de algunos hongos interesantes españoles. Boletín de la Estación Central de Ecología 4(7): 35-49.

Telleria-Jorge, M.T., Moreno, G. \& Calonge, F.D. 1975. Algunos hongos españoles que viven sobre sustrato leñoso. Boletín de la Estación Central de Ecología 4(8): 29-38.

Torre, M. de la \& Calonge, F.D. 1975. Contribución al catálogo de los Myxomycetes de España. I. Anales del Instituto Botánico Antonio José Cavanilles 32 (1): 89-99.

\section{6}

Calonge, F.D., Moreno, G., Torre, M. de la \& Telleria, M.T. 1976. A propósito del "gurumelo". Boletín de la Sociedad Micológica Castellana 1: 18-21.

Calonge, F.D., Ryvarden, L. \& Telleria Jorge, M.T. 1976. Nota sobre los Aphyllophorales de España. I. Lagascalia 6: 7-21.

Calonge, F.D. \& Zugaza, A. 1976. Catálogo de los hongos presentados en la II Exposición de Madrid, del 16 al 18 de Noviembre de 1973. Boletín de la Sociedad Micológica Castellana 1:5-12.

Moreno, G., Calonge, F.D., Telleria, M.T. \& Torre, M. de la. 1976. Contribución al estudio y distribución de Agaricales en la provincia de Madrid. I. Boletaceae Chev. Boletín de la Sociedad Micológica Castellana 1: 30-34.

Ryvarden, L. \& Calonge, F.D. 1976. Type-Studies in the Polyporaceae 5. Species described by Lázaro e Ibiza. Nova Hedwigia 27: 155-164.

Telleria, M.T., Calonge, F.D., Torre, M. de la \& Moreno, G. 1976. Tyromyces fissilis (Bert. \& Curt.) Donk; nuevo para España peninsular. Boletín de la Sociedad Micológica Castellana 1: 27-29.

Torre, M. de la, Calonge, F.D., Moreno, G. \& Telleria, M.T. 1976. La familia Sarcoscyphaceae (Ascomycetes) en España. Boletín de la Sociedad Micológica Castellana 1:35-36.

Torre, M. de la, Moreno, G., Telleria, M.T. \& Calonge, F.D. 1976. Aportación al conocimiento de los hongos pirófilos de España. Boletín de la Estación Cantral de Ecología 5(10): 21-31.

Zugaza, A., Calonge, F.D., Torre, M. de la, Moreno, G. \& Telleria, M.T. 1976. Catálogo de los hongos presentados en la III Exposición de Castilla, del 7 al 9 de Noviembre de 1975. Boletín de la Sociedad Micológica Castellana 1: 13-17. 


\section{7}

Calonge, F.D. 1977. Resumen de un año de actividades de la Sociedad Micológica. Boletín de la Sociedad Micológica Castellana 2: 2-3.

Calonge, F.D. 1977. Elementos fundamentales a tener en cuenta en la clasificación de los Gasteromycetes. Boletín de la Sociedad Micológica Castellana 2: 1822.

Calonge, F.D. \& Torre, M. de la. 1977. Gyromitra tasmanica (Berk.) Berk. \& Cooke, new to Europe. Transations of the British Mycological Society 68: 483-484.

Calonge, F.D., Torre, M. de la \& Ławrynowicz, M. 1977. Contribución al estudio de los hongos hipogeos de España. Anales del Instituto Botánico Antonio José Cavanilles 34(1): 15-31.

Telleria, M.T. \& Calonge, F.D. 1977. El género Phellinus (Aphyllophorales, Basidiomycetes) en España. Anales del Instituto Botánico Antonio José Cavanilles 34(1): 59-70.

Torre, M. de la \& Calonge, F.D. 1977. Contribución al estudio del género Peziza (Dill.) L. ex St. Amans en España. Anales del Instituto Botánico Antonio José Cavanilles 34(1): 33-58.

Zugaza, A., Calonge, F.D., Torre, M. de la, Moreno, G. \& Telleria, M.T. 1977. IV Exposición de hongos de Castilla celebrada del 30 de Octubre al 1 de Noviembre de 1976. Boletín de la Sociedad Micológica Castellana 2: 23-27.

\section{8}

Calonge, F.D. 1978. Hongos. In: C. Sáenz, Polen y esporas. Introducción a la palinología y vocabulario palinológico: 95-97, lam. XIII. Ed. H. Blume. Madrid.

Calonge, F.D. \& Álvarez-Torija, E. 1978. Resumen de las actividades de la Sociedad durante 1977. Boletín de la Sociedad Micológica Castellana 3:3-4.

Calonge, F.D., Moreno, G., Telleria, M.T., Verde, L. \& Âlvarez-Torija, E. 1978. Catálogo de los hongos presentados en la V Exposición Micológica de Castilla celebrada del 30 de Octubre a 1 de Noviembre de 1977. Boletín de la Sociedad Micológica Castellana 3: 12-18.

Calonge, F.D., Telleria, M.T., Verde de Millán, L. \& Torre, M. de la. 1978. Una "estrella de tierra" rara, Geastrum melanocephallum (Czern.) Stan., encontrada por primera vez en España. Boletín de la Sociedad Micológica Castellana 3: 30-32.

Calonge, F.D., Torre, M. de la, Telleria, M.T. \& Verde de Millán, L. 1978. Aportación al catálogo de los hongos del Real Jardín Botánico de Madrid. Boletín de la Estación Central de Ecología 7(13):33-47.
Telleria, M.T., Calonge, F.D. \& Verde de Millán, L. 1978. Contribución al estudio del gen. Albatrellus S.F. Gray emend. Pouz. en España. Boletín de la Sociedad Micológica Castellana 3: 48-53.

Torre, M. de la \& Calonge, F.D. 1978. Fimaria hispanica (Ascomycetes) sp. nov. Anales del Instituto Botánico Antonio José Cavanilles 34(2): 387-392 [1977].

Verde de Millán, L., Telleria, M.T., Calonge, F.D. \& Torre, M. de la. 1978. Introducción al estudio de la familia Tricholomataceae. Boletín de la Sociedad Micológica Castellana 3: 36-47.

\section{9}

Calonge, F.D. 1979. Setas (Hongos). Guía ilustrada. Ed. Mundi-Prensa. Madrid

Calonge, F.D. \& Abella, G. 1979. Physarum sulphureum Alb. \& Schw. (Myxomycetes) especie nueva para España. Boletín de la Sociedad Micológica Castellana 4: 39-42.

Calonge, F.D. \& Álvarez-Torija, E. 1979. Resumen de las actividades de la Sociedad durante 1978. Boletín de la Sociedad Micológica Castellana 4:3-5.

Calonge, F.D.\& Telleria, M.T. 1979. Algunos hongos raros en España. Boletín de la Sociedad Micológica Castellana. 4: 35-38.

Telleria, M.T. \& Calonge, F.D. 1979. Algunos Aphyllophorales que viven sobre los carpóforos de otros hongos. Boletín de la Sociedad Micológica Castellana 4: 30-34.

Verde, L. \& Calonge, F.D. 1979. Callistosporium Sing. (Basidiomycetes), género nuevo para España. Boletim da Sociedade Broteriana, Sér. 2, 53: 89-91.

Wright, J.E., Moreno, G. \& Calonge, F.D. 1979. Tulostoma giovanellae Bres. (Basidiomycetes), nuevo para España peninsular. Boletim da Sociedade Broteriana, Série 2, 53: 93-95.

Zugaza, A., Calonge, F.D., Moreno, G., Telleria, M.T. \& Álvarez, E. 1979. Catálogo de los hongos presentados en la VI Exposición Micológica de Castilla, del 3 al 5 de Noviembre de 1978. Boletín de la Sociedad Micológica Castellana 4: 6-15.

\section{0}

Calonge, F.D. 1980. Notas sobre el ascocarpo de los Erysiphaceae (Ascomycetes). Acta Botanica Malacitana 6: 17-23.

Calonge, F.D. 1980. Notas sobre la nomenclatura de los hongos. Boletín de la Sociedad Micológica Castellana 5: 69-73.

Calonge, F.D. 1980. Miscelánea. Correcciones al volumen 4 (1979). Boletín de la Sociedad Micológica Castellana 5: 85. 
[Diego] Calonge, F. de \& Telleria, M.T. 1980. Introducción al conocimiento de los hongos de Doñana (Huelva, España). Lazaroa 2: 271-326.

Lado, C., Moreno, G., Ortega, A. \& Calonge, F.D. 1980. Estudio sobre Myxomycetes. IV. Provincia de Granada. Boletín de la Sociedad Micológica Castellana 5: 55-68.

Ortega, A. \& Calonge, F.D. 1980. Aportación al estudio de los hongos de Andalucía I. Especies nuevas o interesantes de la provincia de Granada. Acta Botanica Malacitana 6: 83-93.

Ortega, A. \& Calonge, F.D. 1980. Aportación al estudio de los hongos de Andalucía. II. Myxomycetes de la provincia de Granada. Anales del Jardín Botánico de Madrid 36: 9-16 [1979].

Ortega, A. \& Calonge, F.D. 1980. Aportación al estudio de los hongos de Andalucía III. Gasteromycetes de la provincia de Granada. Boletín de la Sociedad Micológica Castellana 5: 25-45.

Verde, L. \& Calonge, F.D. 1980. El género Leucopaxillus Boursier (Basidiomycetes) en España. Boletín de la Sociedad Micológica Castellana 5: 47-54.

\section{1}

Calonge, F.D. 1981. El género Geastrum Pers. ex Pers., en España. Estudio sistemático y descriptivo. Boletín de la Sociedad Micológica Castellana 6: 9-37.

Calonge, F.D. 1981. Contribución de los botánicos españoles a la Sociedade Broteriana. Boletim da Sociedade Broteriana, Série 2, 54: 411-414.

Calonge, F.D. 1981. Reseña bibliográfica de: M.T. Telleria. Contribución al estudio de los Aphyllophorales españoles. Bibliotheca Mycologica, Band 74, Ed. J. Cramer, Vaduz. 1980. Boletín de la Sociedad Micológica Castellana 6: 131-132.

Calonge, F.D. \& Faus, J. 1981. Cuatro especies pirófilas estrictas del género Peziza. Boletín de la Sociedad Micológica Castellana 6: 101-103.

[Diego] Calonge, F. de. 1981. III. Los hongos. In: Enciclopedia Temática de Asturias 1(5): 71-88. Ed. Silverio Cañada. Gijón.

[Diego] Calonge, F. de. 1981. III. Los hongos (continuación). In: Enciclopedia Temática de Asturias 1(6): 89-104. Ed. Silverio Cañada. Gijón.

[Diego] Calonge, F. de. 1981. Conozcamos las setas. Vida Silvestre 37: 40-49.

[Diego] Calonge, F. de \& Telleria, M.T. 1981. Paseo micológico por el Parque Nacional de Doñana. Vida Silvestre 39: 146-153.

Hjortstam, K., Telleria, M.T., Ryvarden, L. \& Calonge, F.D. 1981. Notes on the Aphyllophorales of Spain. II. Nova Hedwigia 34: 525-538.

Torija, M.E. \& Calonge, F.D. 1981. Normas para el comercio interior y exterior de setas frescas y en conserva en España. Boletín de la Sociedad Micológica Castellana 6: 93-100.

\section{2}

Calonge, F.D. 1982. Adiciones al catálogo de hongos hipogeos de España. Garcia de Orta. Série de Estudios Agronómicos 9: 143-146.

Calonge, F.D. 1982. Scleroderma texense Berk. (Gasteromycetes); hongo casi desconocido en Europa. Boletín de la Sociedad Micológica Castellana 7: 89-93.

[Diego] Calonge, F. de. 1982. Un paseo por el Real Jardín Botánico de Madrid. Vida Silvestre 44: 246255.

Honrubia, M., Calonge, F.D., Demoulin,V., Moreno, G. \& Llimona, X. 1982. Aportación al conocimiento de los hongos del SE. de España VI: Esclerodermatales, Licoperdales, Nidulariales, Falales, Himenogasterales, Podoxales (Gasteromicetes, Basidiomicetes). Anales de la Universidad de Murcia 38: 101-132.

López de los Mozos, A.I. \& Calonge, F.D. 1982. Aportación al catálogo micológico de la provincia de Guadalajara. Boletín de la Sociedad Micológica Castellana 7: 95-104.

\section{3}

Calonge, F.D. 1983. El género Scleroderma Pers. (Gasteromycetes) en España. Revista de Biologia (Lisboa) 12: 49-60.

Calonge, F.D. 1983. Algunos hongos raros o nuevos para España. Lazaroa 5: 283-289.

Calonge, F.D., Abella, G. \& Tellería, M.T. 1983. Contribución al conocimiento de los hongos de las comunidades naturales de Guadalajara. In: Memorias del Coloquio de Ecología y Biogeografía. Guadalajara 28 al 31 de marzo 1979: 89-96. Excma. Diputación de Guadalajara. Madrid [1981].

\section{4}

Calonge, F.D. 1984. Adiciones y correcciones al catálogo del género Geastrum en España. Boletín de la Sociedad Micológica Castellana 8: 83-92 [1983].

Calonge, F.D. 1984. Fimaria canina sp. nov. data to identify the species of the genus Fimaria. Boletin de la Sociedad Micológica Castellana 8: 23-27 [1983].

Calonge, F.D. 1984. Reseña bibliográfica de: Setas del País Vasco. 13. ${ }^{a}$ Serie. 1983. Editada por la Sección de Micología de la Sociedad de Ciencias Aranzadi, bajo el patrocinio de la Caja de Ahorros municipal de San Sebastián. Boletín de la Sociedad Micológica Castellana 8: 147-148 [1983].

Calonge, F.D. 1984. Reseña bibliográfica de: Bolets de Catalunya. (Setas de Cataluña) II. Collecció. 1983. Ed. Societat Catalana de Micologia en colabora- 
ción con la Caixa de Barcelona. Boletín de la Sociedad Micológica Castellana 8: 148 [1983].

Calonge, F.D. \& Abella, G. 1984. Nuevos datos para la micoflora de Guadalajara. Boletín de la Sociedad Micológica Castellana 8: 107-112 [1983].

Faus, J. \& Calonge, F.D. 1984. Notas sobre algunos Ascomycetes interesantes encontrados en Cataluña. Boletín de la Sociedad Micológica Castellana 8: 35-41 [1983].

Moreno, G., Honrubia, M., Calonge, F.D. \& Wright, J.E. 1984. Aportación al conocimiento de los hongos del S.E. de España. V. Tulostomatales (Gasteromycetes). Boletín de la Sociedad Micológica Castellana 8: 93-106 [1983].

\section{5}

Calonge, F.D. 1985. El orden Phallales (Gasteromycetes) en España. I. Phallaceae y Clathraceae. Boletín de la Sociedad Micológica Castellana 10: 59-72.

Calonge, F.D. 1985. Bovista ochrotricha Kreisel (Gasteromycetes), segunda cita mundial de esta especie. Boletín de la Sociedad Micológica Castellana 10: 73-74.

Calonge, F.D. 1985. Reseña bibliográfica de: M. Locquin. Mycologie gérérale et structurales. Ed. Masson, Barcelona. 1984. Boletín de la Sociedad Micológica Castellana 10: 122-123.

Calonge, F.D. 1985. Reseña bibliográfica de: G. Lazzari \& F. Bellu. Atlante iconográfico 1981-1983. Supp. Boll. Gr. Micológico G. Bresadola Trento n. ${ }^{\circ}$ 1-2. 1985. Boletín de la Sociedad Micológica Castellana 10: 123.

Calonge, F.D. 1985. Reseña bibliográfica de: Bolets de Catalunya. III Collecció. 1984. Edita Societat Catalana de Micologia en colaboración con la Caixa de Barcelona. Boletín de la Sociedad Micológica Castellana 10: 124.

Calonge, F.D. 1985. Reseña bibliográfica de: Fichas técnicas. II Serie. 1984. Ed. Societat Catalana de Micologia, en colaboración con la Caixa de Barcelona. Boletín de la Sociedad Micológica Castellana 10: 124.

Calonge, F.D. 1985. Reseña bibliográfica de: Setas del País Vasco. 14. ${ }^{a}$ serie. 1984. Edita sección de Micología de la Sociedad de Ciencias Aranzadi, bajo el patrocinio de la Caja de Ahorros Municipal de San Sebastián. Boletín de la Sociedad Micológica Castellana 10: 124-125.

Calonge, F.D. 1985. Reseña bibliográfica de: R. Lotina Benguria. Mil setas ibéricas. 1985. Ed. Diputación Foral de Vizcaya, Bilbao. Boletín de la Sociedad Micológica Castellana 10: 125.

Calonge, F.D. \& Jerez, M. 1985. Poros y otras perforaciones en el capilicio de los Gasteromycetes. Boletín de la Sociedad Micológica Castellana 10: 75-81.
Calonge, F.D., Rocabruna, A. \& Tabarés, M. 1985. Nuevos datos sobre los hongos hipogeos de España. Boletín de la Sociedad Micológica Castellana 9: 45-53 [1984].

Calonge, F.D., Rocabruna, A., Tabarés, M. \& Rodríguez, N.B. 1985. Contribución al estudio de los Ascomicetes españoles. I. Algunas especies nuevas o raras encontradas en Cataluña y Madrid. Butlletí de la Societat Catalana de Micologia 9: 39-47.

Calonge, F.D., Rocabruna, A., Tabarés, M. \& Rodríguez, N.B. 1985. Nuevos datos sobre los hongos hipogeos de España. II. Géneros Balsamia, Delastria y Genea, novedades para el catálogo español. Butlletí de la Societat Catalana de Micologia 9:57-64.

Calonge, F.D. \& Rodríguez, N.B. 1985. Pseudombrophila Boud. (Discomycetes), género nuevo para España. Anales del Jardín Botánico de Madrid 42: 33-37.

[Diego] Calonge, F. de. 1985. La Reserva Biológica de Muniellos (Asturias). Tarrelos 3: 26-28.

[Diego] Calonge, F. de \& Mayoral i Casanovas, A. 1985. Fimaria ripensis (E.C. Hansen) Korf. In: Societat Catalana de Micologia (ed.), Fichas Técnicas, III. ${ }^{a}$ Serie: 50 . Barcelona.

[Diego] Calonge, F. de \& Tabarés Carriedo, M. 1985. Peziza endocarpoides Berk. In: Societat Catalana de Micologia (ed.), Fichas Técnicas, III. ${ }^{a}$ Serie: 65. Barcelona.

[Diego] Calonge, F. de \& Tabarés Carriedo, M. 1985. Plectania melastoma Sov. ex (S.F. Gray) Fuckel. In: Societat Catalana de Micologia (ed.), Fichas Técnicas, III. ${ }^{a}$ Serie: 69 . Barcelona.

Ortega, A., Buendía, A.G. \& Calonge, F.D. 1985. Estudio de algunas especies interesantes del género Lycoperdon (Gasteromycetes) en España. Boletín de la Sociedad Micológica Castellana 9: 141-147 [1984].

Rodríguez, N.B. \& Calonge, F.D. 1985. Nuevos datos para el catálogo de Ascomycetes españoles. Boletín de la Sociedad Micológica Castellana 9: 15-20.

\section{6}

Arroyo, I. \& Calonge, F.D. 1986. Algunos hongos nuevos o poco conocidos para España. Boletín de la Sociedad Micológica de Madrid 11: 111-118.

Calonge, F.D. 1986. Aportación al conocimiento de los hongos pirófilos de España. II. Estudio comparativo de la micoflora de cinco zonas quemadas en la provincia de Ávila. Boletín de la Sociedad Micológica de Madrid 11: 97-110.

Calonge, F.D. 1986. Adiciones al catálogo de hongos que crecen espontáneamente en el Real Jardín Botánico de Madrid. Boletín de la Sociedad Micológica de Madrid 11: 119-124. 
Calonge, F.D. 1986. Resumen de las actividades de la Sociedad durante 1985. Boletín de la Sociedad Micológica de Madrid 11: 168-172.

Calonge, F.D. 1986. Ilustraciones de hongos y líquenes en la Expedición Botánica a Nueva Granada, depositadas en el Real Jardín Botánico de Madrid. Caldasia 15(71-75): 47-51.

Calonge, F.D. 1986. Datos sobre la obra de José Celestino Mutis depositada en el Real Jardín Botánico. In: P. Martín Ferrero (ed.), Actas del Simposio Aniversario del Nacimiento de José Celestino Mutis: 381-383. Diputación Provicial de Cádiz. Cádiz.

Calonge, F.D., Rocabruna, A., Tabarés, M. \& Sierra, M.D. 1986. Contribución al estudio de los Ascomycotina españoles. II. Especies interesantes encontradas en Cataluña. Boletín de la Sociedad Micológica de Madrid 11: 27-37.

Calonge, F.D., Rocabruna, A., Tabarés, M. \& Sierra, M.D. 1986. Contribución al estudio de los Ascomycotina españoles. III. Un género y ocho especies nuevas para el catálogo. Butlletí de la Societat Catalana de Micologia 10:35-46.

Calonge, F.D. \& Rodríguez, N.B. 1986. Nuevos datos para el catálogo de Ascomycetes españoles. II. Registro de un género y siete especies nuevas para España. Boletín de la Sociedad Micológica de Madrid 11: 21-25.

[Diego] Calonge, F. de \& Ławrynowicz, M. 1986. A contribution to the chorology of some Gasteromycetes in Poland. Acta Mycologica 18: 161-169 [1982].

Rodríguez, N.B. \& Calonge, F.D. 1986. Contribución al estudio micológico de la Reserva Biológica de Muniellos (Asturias). Ascomycotina. Revista Ibérica de Micología 3: 5-12.

\section{7}

Calonge, F.D. 1987. Algunas novedades micológicas dignas de interés. Boletín de la Sociedad Micológica de Madrid 11: 253-260 [1986].

Calonge, F.D. 1987. Reseña bibliográfica de: Bolets de Catalunya. IV. Colección. 1985. Edita Societat Catalana de Micologia en colaboración con la Caixa de Barcelona. Boletín de la Sociedad Micológica de Madrid 11: 311 [1986].

Calonge, F.D. 1987. Reseña bibliográfica de: Bolets de Catalunya. V. Colección. 1986. Ed. Societat Catalana de Micologia en colaboración con la Caixa de Barcelona. Boletín de la Sociedad Micológica de Madrid 11:311 [1986].

Calonge, F.D. 1987. Reseña bibliográfica de: Fichas técnicas. 3. a serie. 1985. Ed. Societat Catalana de Micologia en colaboración con la Caixa de Barcelona. Boletín de la Sociedad Micológica de Madrid 11:311-312 [1986].
Calonge, F.D. 1987. Reseña bibliográfica de: Fichas técnicas. 4. ${ }^{a}$ serie. 1986. Ed. Societat Catalana de Micología en colaboración con la Caixa de Barcelona. Boletín de la Sociedad Micológica de Madrid 11: 312 [1986].

Calonge, F.D. 1987. Resumen de las actividades de la Sociedad durante 1986. I. Excursiones micológicas. Boletín de la Sociedad Micológica de Madrid 11: 315-316 [1986].

Calonge, F.D. 1987. Resumen de las actividades de la Sociedad durante 1986. III. Exposición de Hongos. Boletín de la Sociedad Micológica de Madrid 11: 316-319 [1986].

Calonge, F.D. 1987. Contribución al estudio micológico de la Reserva Biológica de Muniellos (Asturias). II. Basidiomycotina. Revista Ibérica de Micología 4: 87-92.

Calonge, F.D. \& Arroyo, I. 1987. Aportación al catálogo micológico de la provincia de Cuenca. Boletín de la Sociedad Micológica de Madrid 11: 241-252 [1986].

Calonge, F.D. \& López-Herce, J.A. 1987. Tres casos de envenamiento grave en Madrid por ingestión de Lepiota brunneo-incarnata Chodat \& Martin. Boletín de la Sociedad Micológica de Madrid 11: 287 290 [1986].

Ladero, M., Calonge, F.D., Valle, C.J., Marcos, B., Santos, M.T., Fernández-Arias, M.I. \& Amor, A. 1987. Aportaciones al conocimiento micológico del centro-oeste español. (I Curso de Micología). Studia Botanica, Salamanca 6: 75-81.

\section{8}

Arroyo, I. \& Calonge, F.D. 1988. Melastiza asperula Spooner, nueva para España, taxon de posición incierta. Boletín de la Sociedad Micológica de Madrid 12: 23-26 [1987].

Arroyo, I., Torre, M. de la \& Calonge, F.D. 1988. Peziza donadiniana sp. nov. from Spain. Transations of the British Mycological Society 90: 132-135.

Calonge, F.D. 1988. Reseña bibliográfica de: C. Constantino Mas \& J. Siquier Virgos. Petita guia dels bolets de les Balears. Ed. Govern Balear, Consellería d'Agricultura i Pesca. Palma de Mallorca, 1985. Boletín de la Sociedad Micológica de Madrid 12: 137-138 [1987].

Calonge, F.D. 1988. Reseña bibliográfica de: M. García Rollán. Cultivo de setas y trufas. Ed. Mundi-Prensa, Madrid 1987. Boletín de la Sociedad Micológica de Madrid 12: 138 [1987]

Calonge, F.D. 1988. Reseña bibliográfica de: M. García Rollán. Setas de los árboles (Hongos de la madera). 2. ${ }^{a}$ edición. Publicaciones de Extensión Agraria, Madrid 1984. Boletín de la Sociedad Micológica de Madrid 12: 138 [1987]. 
Calonge, F.D. 1988. Reseña bibliográfica de: R. Mendaza Rincón de Acuña \& G. Díaz Montoya. Las Setas. Guía fotográfica y descriptiva. 800 especies a todo color. Sección de Micología de Iberduero. Sondica, Vizcaya, 1987. Boletín de la Sociedad Micológica de Madrid 12: 139-141 [1987].

Calonge, F.D. 1988. Reseña bibliográfica de: Setas del País Vasco. 16. ${ }^{a}$ serie. 1986. Sección de Micología de la Sociedad de Ciencias Naturales Aranzadi, Caja de Ahorros Municipal de San Sebastián. Boletín de la Sociedad Micológica de Madrid 12: 141 [1987].

Calonge, F.D. 1988. Reseña bibliográfica de: J.R. Gil \& P. Die. Guía de setas de Extremadura. Ediciones Fondo Natural. Madrid 1987. Boletín de la Sociedad Micológica de Madrid 12: 141-142 [1987].

Calonge, F.D. 1988. Reseña bibliográfica de: Bolets de Catalunya. VI Colección. 1987. Ed. Societat Catalana de Micologia en colaboración con la Caixa de Barcelona. Boletín de la Sociedad Micológica de Madrid 12: 142 [1987].

Calonge, F.D. 1988. Reseña bibliográfica de: Fichas técnicas. 5. ${ }^{a}$ Serie. 1987. Ed. Societat Catalana de Micologia en colaboración con la Caixa de Barcelona. Boletín de la Sociedad Micológica de Madrid 12: 142-143 [1987].

Calonge, F.D. 1988. Reseña bibliográfica de: D. Sierra López. Aportación al conocimiento de los Ascomicetes (Ascomycotina) de Cataluña. Ed. Societat Catalana de Micologia, vol. 1. Barcelona 1987. Boletín de la Sociedad Micológica de Madrid 12: 143 [1987].

Calonge, F.D. 1988. Reseña bibliográfica de: M.L. Castro \& L. Freire. Guia das setas ou cogumelos comestibles de Galicia. Ed. Xerais de Galicia, S. A. Vigo, 1982. Boletín de la Sociedad Micológica de Madrid 12: 143-144 [1987].

Calonge, F.D. 1988. Resumen de las actividades de la Sociedad durante 1987. I. Excursiones micológicas. Boletín de la Sociedad Micológica de Madrid 12: 145-146 [1987].

Calonge, F.D. 1988. Resumen de las actividades de la Sociedad durante 1987. III. Exposición de hongos. Boletín de la Sociedad Micológica de Madrid 12: 147 [1987].

Calonge, F.D. 1988. Dos casos de "envenenamiento" leve en Madrid por ingestión de setas habitualmente comestibles. Boletín de la Sociedad Micológica de Madrid 12: 154-156 [1987].

Calonge, F.D. 1988. Bovista bovistoides (Cooke \& Massee) Ahmad, Gasteromycetes, Fungi, nueva para Europa. Monografías del Instituto Pirenaico de Ecología 4: 127-129 [1987].
Calonge, F.D. 1988. Taxonomy of truffles. Atti del II Congreso Internazionale sul Tartufo, Spoleto: 35-36.

Calonge, F.D. 1988. La coltivazione dei funghi commestibili in Spagna. Mushroom Information. La Rivista del Fungicoltore Moderno anno V, N. 3/4: sin paginar.

Calonge, F.D., Donadini, J.C., Torre, M. de la, Rocabruna, A. \& Tabarés, M. 1988. Trichophaea paraphysincrustata (Ascomycotina), especie nueva para la ciencia. Boletín de la Sociedad Micológica de Madrid 12: 27-33 [1987].

Calonge, F.D. \& Oria de Rueda, J.A. 1988. Aportación a la micoflora de la provincia de Almería. Boletín de la Sociedad Micológica de Madrid 12: 93-106 [1987].

Calonge, F.D. \& Palmer, J.T. 1988. Mycocalia denudata (Fr.) J.T. Palmer, nueva para España. Boletín de la Sociedad Micológica de Madrid 12: 131-132 [1987].

Demoulin,V., Martín, M.P. \& Calonge, F.D. 1988. Calvatia pachyderma (Peck) Morgan (Gasteromycetes), nueva para Europa. Boletín de la Sociedad Micológica de Madrid 12: 85-88 [1987].

[Diego] Calonge, F. de. 1988. El bosque y sus hongos. Plus Ultra. Madrid.

Torre, M. de la, Rocabruna, A., Tabarés, M., Arroyo, I. \& Calonge, F.D. 1988. Contribución al estudio de Ascomycotina españoles. IV. Algunas especies interesantes de Cataluña. Boletín de la Sociedad Micológica de Madrid 12: 35-38 [1987].

\section{9}

Arroyo, I. \& Calonge, F.D. 1989. A new species of Peziza in Spain: P. barahonae. Cryptogamic Botany 1: 80-82.

Arroyo, I., Calonge, F.D., Carrascosa, G. \& Sau, E. 1989. Nuovi funghi ipogei di Spagna III. Hydnocystis clausa (Tul.) Ceruti e Genea verrucosa Vitt. Micologia Italiana 18: 3-7.

Calonge, F.D. 1989. Resumen de las actividades de la Sociedad durante 1988. Boletín de la Sociedad Micológica de Madrid 13: 213-219 [1988].

Calonge, F.D. 1989. Cenosis fúngica en la España mediterránea. Micologia e Vegetazione Mediterranea 4(2): 21-28.

Calonge, F.D. \& Guerra, A. 1989. Helicobasidium brebissonii (Desm.) Donk, Auriculariales, un hongo curioso. Boletín de la Sociedad Micológica de Madrid 13: 197-199 [1988].

Calonge, F.D. \& Romero Zarco, C. 1989. Ileodictyon gracile Berk. (Gasteromycetes), nuevo para el catálogo micológico español. Boletín de la Sociedad Micológica de Madrid 13: 171-174 [1988].

Calonge, F.D., Siquier, J.L., Constantino, C. \& Lillo, X. 1989. Contribución al conocimiento micológico 
de las Islas Baleares. I. Gasteromycetes. Boletín de la Sociedad Micológica de Madrid 13: 139-150 [1988].

Calonge, F.D. \& Vidal, J.M. 1989. Hysterangium inflatum Rodw. (Gasteromycetes), nuevo para España. Boletín de la Sociedad Micológica de Madrid 13: 201-203 [1988].

Calonge, F.D. \& Wright, J.E. 1989. El género Tulostoma Pers.: Pers. (Gasteromycetes) en España. Boletín de la Sociedad Micológica de Madrid 13: 119. 137 [1988].

[Diego] Calonge, F. de. 1989. El Real Jardín Botánico de Madrid. In: Aula de Cultura. Ciclo de conferencias: el Madrid de Carlos III, n. ${ }^{\circ} 24$. Ayuntamiento de Madrid \& Instituto de Estudios Madrileños (CSIC). Madrid.

Ipinza Carmona, R., Martínez de Azagra, A. \& Calonge, F.D. 1989. Consideraciones micológicas sobre Ceratocystis (Ophiostoma) ulmi (Buism.) Moreau, como base para el control de la grafiosis del olmo. Boletín de la Sociedad Micológica de Madrid 13: 4987 [1988].

\section{0}

Arroyo, I. \& Calonge, F.D. 1990. Pseudombrophila misturae (Phill.) Svrcek y Helvella leucopus Pers. var. populina Arroyo \& Calonge, nuevas para España. Boletín de la Sociedad Micológica de Madrid 14: 197-199 [1989].

Arroyo, I., Calonge, F.D., Siquier, J.L. \& Constantino, C. 1990. Contribución al conocimiento micológico de las Islas Baleares, II. Ascomycotina. Boletín de la Sociedad Micológica de Madrid 14: 49-60 [1989].

Calonge, F.D. 1990. Algunos Gasteromycetes interesantes de Extremadura. Boletín de la Sociedad Micológica de Madrid 14: 191-195 [1989].

Calonge, F.D. 1990. Adiciones al catálogo de los Gasteromycetes de España. I. Registro de dos nuevas especies: Geastrum pseudolimbatum Hollós y Mycocalia duriaeana (Tul.) J.T. Palmer. Boletín de la Sociedad Micológica de Madrid 14: 201-203 [1989].

Calonge, F.D. 1990. Check-List of the Spanish Gasteromycetes (Fungi, Basidiomycotina). Cryptogamic Botany 2: 33-55.

Calonge, F.D. 1990. Setas (Hongos). Guía ilustrada. 2. ed. Ed. Mundi-Prensa. Madrid.

Calonge, F.D. 1990. Reseña bibliográfica de: S. Santamaría. El orden Laboulbeniales (Fungi, Ascomycotina) en la Península Ibérica. Ed. Societat Catalana de Micologia, vol. 3. Esplugues, 1989. Boletín de la Sociedad Micológica de Madrid 14: 205 [1989]

Calonge, F.D. 1990. Reseña bibliográfica de: J.R. Gil \& P. Die. Guía de setas de Extremadura. II. Ed.
Fondo Natural. Madrid, 1989. Boletín de la Sociedad Micológica de Madrid 14: 205 [1989].

Calonge, F.D. 1990. Reseña bibliográfica de: E. Ibáñez, I. Menes, J. Arias \& S. Álvarez. Las setas en Asturias. Ed. González \& Huici, S. A. Gijón, 1989. Boletín de la Sociedad Micológica de Madrid 14: 206 [1989].

Calonge, F.D. 1990. Reseña bibliográfica de: L.M. García Bona. Setas y hongos de la Península Ibérica, vols. I, II. Ed. Kriselu, S. A. San Sebastián, 1989. Boletín de la Sociedad Micológica de Madrid 14: 206-207 [1989].

Calonge, F.D. 1990. Reseña bibliográfica de: J. Meléndez Latorre. Setas de Aragón. Ed. Mira, S. A. Zaragoza, 1988. Boletín de la Sociedad Micológica de Madrid 14: 207-208 [1989].

Calonge, F.D. 1990. Reseña bibliográfica de: C. Ortega Hernández-Agero. El Libro rojo de los bosques españoles. ADENA-WWF España, Arroyomolinos (Madrid). 1989. Boletín de la Sociedad Micológica de Madrid 14: 210-211 [1989].

Calonge, F.D., \& Arroyo, I. 1990. Notes on the genus Helvella in Spain. Mycotaxon 39: 203-217.

Calonge, F.D., Carrascosa, G. \& Sau, E. 1990. Contribución al estudio de Ascomycotina españoles. V. Sobre cuatro especies encontradas en Cataluña. Boletín de la Sociedad Micológica de Madrid 14: 4348 [1989].

Calonge, F.D. \& Martín, M.P. 1990. Notes on the taxonomical delimitation in the genera Calvatia, Gastropila and Langermannia (Gasteromycetes). Boletín de la Sociedad Micológica de Madrid 14: 181-190 [1989].

Ipinza Carmona, R., Martínez de Azagra, A., Salvador Nemoz, M.L. \& Calonge, F.D. 1990. Consideraciones micológicas y epidemiológicas de Ceratocystis (Ophiostoma) ulmi (Buism.) Noreav. In: Gil, L. (ed.), Los olmos y la grafiosis en España. Colección Técnica.

\section{1}

Caballero Moreno, A. \& Calonge, F.D. 1991. Leucoagaricus fuligineodiffractus Bellú \& Lanzoni, una posible novedad para España. Boletín de la Sociedad Micológica de Madrid 15: 203-205 [1990].

Calonge, F.D. 1991. Fungi mediterranei rariores 1. Amanita ponderosa. Micologia e Vegetazione Mediterranea 5(1-2): lámina sin paginación.

Calonge, F.D. 1991. Fungi mediterranei rariores. 2. Collybia konradiana. Micologia e Vegetazione Mediterranea 5(1-2): lámina sin paginación.

Calonge, F.D. 1991. Fungi mediterranei rariores 3. Colus hirudinosus. Micologia e Vegetazione Mediterranea 5(1-2): lámina sin paginación. 
Calonge, F.D. 1991. Fungi mediterranei rariores 4. Ileodictyon gracile. Micologia e Vegetazione Mediterranea 6: 39-40.

Calonge, F.D. 1991. Reseña bibliográfica de: Pando, F., Dueñas, M., Lado, C. \& Telleria, M.T. (eds.), Cuadernos de trabajo de Flora Micológica Ibérica 1. Información bibliográfica. I. España peninsular e Islas Baleares. Madrid, 1990. Anales del Jardín Botánico de Madrid 48: 101 [1990].

Calonge, F.D. 1991. Terfecia claveryi Chatin (Ascomycotina) en las Islas Canarias. Boletín de la Sociedad Micológica de Madrid 15: 193-196 [1990].

Calonge, F.D. 1991. Acerca de dos especies curiosas de hongos encontradas recientemente en Extremadura. Boletín de la Sociedad Micológica Extremeña 2: 50-52.

Calonge, F.D. 1991. Ascomycetes y Gasteromycetes. In: Calonge, F.D. (ed.), Las setas de la Comunidad de Madrid: 155-257. Agencia de Medio Ambiente, Comunidad de Madrid. Madrid.

Calonge, F.D., Carrascosa, G. \& Sau, E. 1991. Notas sobre algunos hongos de Cataluña. Boletín de la Sociedad Micológica de Madrid 15: 183-187 [1990].

Calonge, F.D., Dominicis, V. de, Barluzzi, C. \& Perini, C. 1991. Geastrum pseudolimbatum Hollo's and G. saccatum (Fr.) E. Fischer (gasteromyceces) in Italy. Boletín de la Sociedad Micológica de Madrid 15: 177-181 [1990].

Calonge, F.D. \& Martín, M.P. 1991. Notas sobre los Gasteromycetes del Parque Nacional de Ordesa y Monte Perdido (Huesca, España). Boletín de la Sociedad Micológica de Madrid 15: 197-201 [1990].

Calonge, F.D. \& Sánchez, A. 1991. Bovistella radicata (Gasteromycetes) en Extremadura, España. Boletín de la Sociedad Micológica de Madrid 15: $207-$ 208 [1990].

Calonge, F.D., Siquier, J.L. \& Constantino, C. 1991. Contribución al conocimiento micológico de las Islas Baleares, III. Bovista cunninghamii Kreisel y Tuber uncinatum Chatin, dos nuevas citas para el catálogo español. Boletín de la Sociedad Micológica de Madrid 15: 111-122 [1990].

\section{2}

Calonge, F.D. 1992. Nuevos datos sobre el género Tulostoma (Gasteromycetes) en España. Tulostoma bruchi Spegaz., nuevo para Europa. Boletín de la Sociedad Micológica de Madrid 16: 149-153 [1991].

Calonge, F.D. 1992. Reseña bibliográfica de: Societat Catalana de Micologia (ed.). Bolets de Catalunya. X Collecció. Esplugues de Llobregat, 1991. Boletín de la Sociedad Micológica de Madrid 16: 179 [1991].

Calonge, F.D. 1992. El género Bovista Pers.: Pers. (Gasteromycetes), en la Península Ibérica e Islas
Baleares. Boletín de la Sociedad Micológica de Madrid 17: 101-113.

Calonge, F.D. 1992. Nomenclatura y taxonomía de los hongos: el caos permanente. Boletín de la Sociedad Micológica de Madrid 17: 167-175.

Calonge, F.D. 1992. ¿Puede producir trastornos la Morchella esculenta var. umbrina? Boletín de la Sociedad Micológica de Madrid 17: 204-205.

Calonge, F.D. 1992. Los hongos. In: Guía de la naturaleza de Galicia 22: 421-440. El Faro de Vigo. Pontevedra.

Calonge, F.D. 1992. Tulostoma bruchi (Gasteromycetes, Basidiomycotina), una especie argentina introducida en Europa. Boletin de la Sociedad Argentina de Botánica 28: 247-248.

Calonge, F.D. \& Almeida, M.G. 1992. Catálogo de los Gasteromycetes de Portugal. Boletín de la Sociedad Micológica de Madrid 16: 73-108 [1991].

Calonge, F.D. \& Almeida, M.G. 1992. Tulostoma lusitanicum sp. nov. (Gasteromycetes). Boletín de la Sociedad Micológica de Madrid 16: 109-113 [1991].

Calonge, F.D. \& Arroyo, I. 1992. Helvella spadicea Schaeffer, nombre correcto para Helvella leucopus Persoon, Ascomycotina. Boletín de la Sociedad Micológica de Madrid 16: 141 [1991]

Calonge, F.D., Caballero, A. \& Palacios, J. 1992. Contribución al conocimiento de los hongos de $\mathrm{La}$ Rioja (Logroño, España). Gasteromycetes. Boletín de la Sociedad Micológica de Madrid 16: 115-140 [1991].

Calonge, F.D., Carrascosa, G. \& Sau, E. 1992. Notas sobre algunos hongos de Cataluña pertenecientes a los Ascomycotina y Gasteromycetes. Boletín de la Sociedad Micológica de Madrid 16: 143-147 [1991].

Calonge, F.D., Gil, J.R. \& Fernández, I. 1992. Phallus rubicundus (Bosc) Fr. (Gasteromycetes) encontrado en Extremadura (España), nuevo para Europa. Boletín de la Sociedad Micológica de Madrid 16: 165-169 [1991].

Calonge, F.D., González, J.A., Andrés, J. \& Sánchez, J.A. 1992. Catálogo provisional de los Gasteromycetes de León. Boletín de la Sociedad Micológica de Madrid 17: 123-136.

Calonge, F.D., Guerra, A. \& Jerez, M. 1992. Notas sobre algunas "Criadillas de Tierra". Boletín de la Sociedad Micológica Extremeña 3: 33-36.

Calonge, F.D. \& Marcos, B. 1992. Una variedad nueva de Lysurus cruciatus (Lepr. \& Mont.) Lloyd. Boletín de la Sociedad Micológica de Madrid 16: 155157 [1991].

Calonge, F.D., Marcos, B., Valle, C.J. \& Lozano, J. 1992. Aportaciones corológico-taxonómicas sobre algunos hongos poco frecuentes en España. Boletín 
de la Sociedad Micológica de Madrid 16: 159-163 [1991].

Calonge, F.D. \& Martín, M.P. 1992. Nuevos datos sobre el género Tulostoma (Gasteromycetes) en España. II. Tulostoma xerophilum, nuevo para Europa. Boletín de la Sociedad Micológica de Madrid 17: 115-122.

Calonge, F.D. \& Pasabán, P.M. 1992. Nuevos datos sobre los hongos hipogeos de España. IV Gymnomyces xanthosporus (Hawker) A.H. Smith, novedad para el catálogo español. Boletín de la Sociedad Micológica de Madrid 16: 29-45 [1991].

Calonge, F.D. \& Pegler, D.N. 1992. Rickenella mellea in Antarctica. The Mycologist 6: 174-175.

Calonge, F.D., Rocabruna, A. \& Tabarés, M. 1992. Phallogater saccatus Morgan (Gasteromycetes), nuevo para la Península Ibérica. Boletín de la Sociedad Micológica de Madrid 17: 177-179.

Calonge, F.D., Siquier, J.L. \& Constantino, C. 1992. Contribución al conocimiento micológico de las Islas Baleares. IV. Adiciones al catálogo de Gasteromycetes. Boletín de la Sociedad Micológica de Madrid 16: 61-71 [1991].

Calonge, F.D. \& Tomo, A.P. 1992. Omphalina ballesteri (Basidiomycotina), a new species from the Antarctica. Boletín de la Sociedad Micológica de Madrid 16: 42-52 [1991].

Campoamor, J.N. \& Calonge, F.D. 1992. Estudios sobre Tricholomataceae ibéricos. I. Collybia prolixa y C. tuberosa, dos especies interesantes para nuestro catálogo. Boletín de la Sociedad Micológica de Madrid 17: 57-61.

Peláez, F., Díez, M.T. \& Calonge, F.D. 1992. Obtención de cultivos puros de Basidiomycotina a partir de carpóforos recogidos en España. Boletín de la Sociedad Micológica de Madrid 17: 153-165.

\section{3}

Calonge, F.D. 1993. Hacía la confección de una lista roja de Macromycetes (hongos) en la Península Ibérica. Boletín de la Sociedad Micológica de Madrid 18: 171-178.

Calonge, F.D. 1993. Hongos medicinales. Boletín de la Sociedad Micológica de Madrid 18: 179-188.

Calonge, F.D. 1993. Endoptychum agaricoides y Torrendia pulchella. Dos hongos interesantes presentes en Extremadura. Boletín de la Sociedad Micológica Extremeña 4: 32-35.

Calonge, F.D., Carrascosa, G. \& Estanyol, S. 1993. Bovista ochrotricha Kreisel, nueva cita para España peninsular. Boletín de la Sociedad Micológica de Madrid 18: 189-191.

Calonge, F.D. \& Pasabán, P.M. 1993. Nuevos datos sobre los hongos hipogeos de España. V. Registro de nueve citas nuevas. Boletín de la Sociedad Micológica de Madrid 18: 41-57.

Calonge, F.D., Santos, J.C. \& García, F. 1993. Contribución al estudio de los hongos de Valladolid y provincias limítrofes. Gasteromycetes y Ascomycetes hipogeos. Boletín de la Sociedad Micológica de Madrid 18: 59-79.

Calonge, F.D., Siquier, J.L. \& Constantino, C. 1993. Contribución al conocimiento micológico de las Islas Baleares. V. Registro de trece citas nuevas. Boletín de la Sociedad Micológica de Madrid 18: $105-$ 115.

Calonge, F.D., Terrón, A., Pérez Jarauta, T. \& López Mariño, A. 1993. Algunos hongos hipogeos de León, Soria y Jaén. Boletín de la Sociedad Micológica de Madrid 18: 81-85.

Castro, M.L., Freire, L. \& Calonge, F.D. 1993. Catálogo provisional de los Gasteromycetes de Galicia (España). Boletín de la Sociedad Micológica de Madrid 18: 87-104.

Kreisel, H. \& Calonge, F.D. 1993. Calvatiella Chow., a synonym for Bovistella Morgan. Mycotaxon 48: 13-23.

\section{4}

Calonge, F.D. 1994. El polémico despertar de hongos prehistóricos. ABC de la Ciencia (22/04/1994): 5153.

Calonge, F.D. 1994. Cyathus stercoreus forma ephedrae, una forma nueva. Boletín de la Sociedad Micológica de Madrid 19:33-36.

Calonge, F.D., Gómez, J. \& Moreno, B. 1994. Gymnomyces ferruginascens Singer \& Smith (Gasteromycetes) en España. Boletín de la Sociedad Micológica de Madrid 19: 305-306.

Calonge, F.D. \& Lanconelli, L. 1994. Radiigera atrogleba Zeller en Italia. Un hongo americano casi desconocido en Europa. Boletín de la Sociedad Micológica de Madrid 19: 87-90.

Calonge, F.D., Mahiques, R. \& Tejedor, F. 1994. Aportación al conocimiento de los hongos de la Comunidad Valenciana (España). Disciseda anomala, una especie nueva para Europa. Boletín de la Sociedad Micológica de Madrid 19: 155-163.

Calonge, F.D. \& Pasabán, P.M. 1994. Hysterangium rubricatum y Leucogaster tozzianus (Gasteromycetes), dos hongos raros en España. Boletín de la Sociedad Micológica de Madrid 19:315-316.

Calonge, F.D., Pérez Jarauta, T., Terrón, A. \& González Cuenca, J.A. 1994. Nuevos datos sobre los hongos hipogeos de España. VI. Gautieria otthii e Hysterangium cistophilum, novedades para el catálogo. Boletín de la Sociedad Micológica de Madrid 19: 165-173. 
Calonge, F.D. \& Ruíz Leivas, C. 1994. Una forma atípica de Morchella esculenta hallada en Galicia. Boletín de la Sociedad Micológica de Madrid 19: $307-$ 308.

Calonge, F.D., Santos, J.C., Juste, P. \& García, F. 1994. Contribución al estudio de los hongos de Valladolid y provincias limítrofes. II. Registro de cuatro táxones nuevos para el catálogo español. Boletín de la Sociedad Micológica de Madrid 19: 175-185.

Castro, A., Infante, F., Gómez-Arjona, J. \& Calonge, F.D. 1994. Contribución al conocimiento micológico de Andalucía. Catálogo de los Gasteromycetes de Córdoba (España). Boletín de la Sociedad Micológica de Madrid 19: 91-109.

[Diego] Calonge, F. de, Jiménez Antonio, F., Guerra de la Cruz, A., Fernández López, C., Briones Pancorbo, P., Cuesta Braceros, M.J., Guerrero Rodríguez, P., Hornos Damas, J.L., Jiménez Herrera, I. \& Guijosa Pulido, M.J. 1994. Setas de otoño en Jaén. Año 1993. Lactarius 3: 26-37.

Martín, M.P. \& Calonge, F.D. 1994. Nuevos datos sobre el género Tulostoma (Gasteromycetes) en España. III. Butlletí de la Societat Catalana de Micologia 16-17: 205-212.

\section{5}

Calonge, F.D. 1995. La Botánica Criptogámica en España. Studia Botanica, Salamanca 13: 15-20 [1994].

Calonge, F.D. 1995. Two secotioid fungi recently found in Spain. Documents Mycologiques 25(98100): 113-118.

Calonge, F.D. \& Equipo de Campo de la Sociedad Vallisoletana de Micología. 1995. Galeropsis desertorum var. bispora encontrada en Valladolid (España). Boletín de la Sociedad Micológica de Madrid 20: 317-319.

Calonge, F.D., García, F., Santos, J.C. \& Juste, P. 1995. Contribución al estudio de los hongos de Valladolid y provincias limítrofes. III. Algunas especies hipogeas interesantes. Boletín de la Sociedad Micológica de Madrid 20: 291-299.

Calonge, F.D., Moreno, B. \& Gómez, J. 1995. Nuevos datos sobre los hongos de Andalucía. Boletín de la Sociedad Micológica de Madrid 20: 283-290.

Calonge, F.D. \& Pasabán, P.M. 1995. Rhodogaster chilensis E. Horak (Gasteromycetes), encontrado en España y nuevo para Europa. Boletín de la Sociedad Micológica de Madrid 20: 277-281.

Calonge, F.D., Rubio, E. \& Jerez, M. 1995. Setchelliogaster tenuipes (Gasteromycetes), segunda cita para la Península Ibérica. Boletin de la Sociedad Micológica de Madrid 20: 305-307.

Calonge, F.D., Siquier, J.L. \& Constantino, C. 1995. Contribución al conocimiento micológico de las Is- las Baleares. VII. Cuatro especies dignas de mención. Boletín de la Sociedad Micológica de Madrid 20: 309-312.

Suárez Álvarez, J.R., Suárez Gonzalo, L., Álvarez Menéndez, M.D. \& [Diego] Calonge, F. de. 1995. Taxonomía práctica para médicos. Sinonimias autonómicas. Revista Clínica Española 195: 256-266.

\section{6}

Calonge, F.D. 1996. Nidularia deformis (Gasteromycetes), una especie rara en la Península Ibérica. Boletín de la Sociedad Micológica de Madrid 21: 395 396.

Calonge, F.D. 1996. Claves de identificación de los Gasteromycetes epigeos ibéricos. Boletín de la Sociedad Micológica de Madrid 21: 359-373.

Calonge, F.D. 1996. Bases corológicas de Flora Micológica Ibérica. Números 693-894. In: Almaraz, T. (ed.), Cuadernos de Trabajo de Flora Micológica 9: 3-240.

Calonge, F.D., Juste, P., García, F. \& Santos, J.C. 1996. Nuevos datos sobre los hongos hipogeos de España. VII. Genea hispidula, novedad para el catálogo. Boletín de la Sociedad Micológica de Madrid 21:325-332.

Calonge, F.D., Núñez, M. \& Ryvarden, L. 1996. Nuevos datos sobre los Aphyllophorales de Venezuela. Boletín de la Sociedad Micológica de Madrid 21: 381-391.

Calonge, F.D. \& Pasabán, P.M. 1996. Contribución al conocimiento de los hongos hipogeos de Navarra. Boletín de la Sociedad Micológica de Madrid 21: 299-303.

Calonge, F.D., Tejedor, F. \& Mahiques, R. 1996. Notas sobre los hongos hipogeos de Castellón. Boletín de la Sociedad Micológica de Madrid 21: 409-411.

Calonge, F.D. \& Verde, L. 1996. Nuevos datos sobre los Gasteromycetes de Venezuela. Boletín de la Sociedad Micológica de Madrid 21: 201-217.

Esteve-Raventós, F. \& Calonge, F.D. 1996. Dos agaricales poco frecuentes e interesantes en la Península Ibérica. Boletín de la Sociedad Micológica de Madrid 21: 293-298.

Kreisel, H. \& Calonge, F.D. 1996. Bovista septina (C.G. Lloyd) Kreisel \& Calonge, comb. nov. Boletín de la Sociedad Micológica de Madrid 21: 393 394.

Moreno-Arroyo, B., Gómez, J. \& Calonge, F.D. 1996. Pachyphloeus prieguensis, sp. nov. (Ascomycotina), encontrada en España. Boletín de la Sociedad Micológica de Madrid 21: 85-92.

Vidal, J.M. \& Calonge, F.D. 1996. Lycoperdon atrum Pat. (Gasteromycetes) nuevo para España. Boletín de la Sociedad Micológica de Madrid 21: 375-379. 


\section{7}

Calonge, F.D. 1997. Some aspects on the ecology and taxonomy of the Iberian Peninsula (Spain and Portugal) Gasteromycetes. Mycologia e Vegetazione Mediterranea 11(2): 115-128 [1996].

Calonge, F.D. 1997. Notes on the genera Pyrenogaster and Radiigera (Gasteromycetes). Boletín de la Sociedad Micológica de Madrid 22: 105-112.

Calonge, F.D. 1997. El género Phallus (Gasteromycetes) en Extremadura, España. Boletín de la Sociedad Micológica Extremeña 8: 53-55.

Calonge, F.D. 1997. Veinticinco años de actividades de la Sociedad Micológica de Madrid (Sociedad Micológica Castellana). Boletín de la Sociedad Micológica de Madrid 22: 3-6.

Calonge, F.D., Harkönen, M., Sarimäki, T. \& Mwasumbi, L. 1997. Tanzanian mushrooms and their uses. 5. Some notes on the Gasteromycetes. Karstenia 37: 3-10.

Calonge, F.D. \& Romero de la Osa, L. 1997. Podoscypha multizonata (Berk. \& Br.) Pat., Aphyllophorales, un hongo casi desconocido en España. Boletín de la Sociedad Micológica de Madrid 22: 295-296.

Moreno-Arroyo, B., Gómez, J., Barba, C.J. \& Calonge, F.D. 1997. Métodos faunísticos para la búsqueda de hongos hipogeos, con especial mención a la especie canina (Canis familiaris). Boletín de la Sociedad Micológica de Madrid 22: 97-103.

Moreno-Arroyo, B., Gómez, J. \& Calonge, F.D. 1997. Aportaciones a la micoflora hipogea ibérica. Boletín de la Sociedad Micológica de Madrid 22: 91-95.

Núñez, M.P. \& Calonge, F.D. 1997. Nuevos datos sobre los Aphyllophorales y Gasteromycetes de Costa Rica. Boletín de la Sociedad Micológica de Madrid 22: 55-59.

Pegler, D.N. \& Calonge, F.D. 1997. Notes on some agaricoid fungi from Venezuela. Boletín de la Sociedad Micológica de Madrid 22: 45-54.

\section{8}

Calonge, F.D. 1998. Notes on five species described by Bresadola. Geographical distribution in the Iberian Peninsula. Bolletino del Gruppo Micologico G. Bresadola, Nuova Serie 40: 133-140 [1997].

Calonge, F.D. 1998. Setas de Madrid. Consejería de Medio Ambiente y Desarrollo Regional. Comunidad de Madrid. Madrid.

Calonge, F.D. 1998. Gasteromycetes, I. Lycoperdales, Nidulariales, Phallales, Sclerodermatales, Tulostomatales. Flora Mycologica Iberica 3: 1-271. Ed. J. Cramer \& Real Jardín Botánico, CSIC.

Calonge, F.D. \& Danniëls, P.P. 1998. Fungi from the Dja Biosphere Reserve (Cameroon). Notes on some Gasteromycetes. Boletín de la Sociedad Micológica de Madrid 23: 171-174.

Calonge, F.D. \& Esteve-Raventós, F. 1998. Agaricus guachari, sp. nov. (Agaricales), from Venezuela. Boletín de la Sociedad Micológica de Madrid 23: 111118.

Calonge, F.D., García, F., Juste, P. \& Santos, J.C. 1998. Nuevos datos sobre los hongos hipogeos de España. VIII. Pyrenogaster pityophilus, segunda cita mundial. Boletín de la Sociedad Micológica de Madrid 23: 215-222.

Calonge, F.D. \& Pegler, D.N. 1998. Zelleromyces hispanicus sp. nov. (Russulales, Elasmomycetaceae), an orange-red species possibly related to Lactarius aurantiacus. Cryptogamie, Mycologie 19: 99-105.

Calonge, F.D. \& Siquier, J.L. 1998. Alpova pseudostipitatus, sp. nov. (Gasteromycetes) from Majorca (Spain) Boletín de la Sociedad Micológica de Madrid 23: 91-96.

Campoamor, J.N. \& Calonge, F.D. 1998. Syzygospora tumefaciens (Ginns \& Sunhede) Ginns (Tremellales), especie nueva para la Península Ibérica. Boletín de la Sociedad Micológica de Madrid 23: 195-198.

Moreno-Arroyo, B., Gómez, J. \& Calonge, F.D. 1998. Zelleromyces giennensis sp. nov. (Russulales), a gasteroid fungus from the South of Spain. Cryptogamie, Mycologie 19: 107-111.

Moreno-Arroyo, B., Gómez, J. \& Calonge, F.D. 1998. Genea subbaetica, sp. nov., from Spain. Boletín de la Sociedad Micológica de Madrid 23: 85-89.

Moreno-Arroyo, B., Gómez, J. \& Calonge, F.D. 1998. Zelleromyces meridionalis (Russulales, Elasmomycetaceae) a new species from Spain. Mycotaxon 69: 467-471.

Moreno-Arroyo, B., Gómez, J. \& Calonge, F.D. 1998. Genea sphaerica f.ma lobulata, f.ma nova dalla Spagna. Bolletino del Gruppo Micologico G. Bresadola, Nuova Serie 41: 205-210.

Oltra, M., Pando, F. \& Calonge, F.D. 1998. Adiciones al catálogo micológico de la Comunidad Valenciana: Myxomycetes y Gasteromycetes. Boletín de la Sociedad Micológica de Madrid 23: 187-194.

\section{9}

Calonge, F.D. 1999. Notas sobre Pyrenogaster pityophilus Malençon \& Riousset (Gasteromycetes, Geastraceae). Boletín de la Sociedad Micológica de Madrid 24: 217-220.

Calonge, F.D. \& Daniëls, P.P. 1999. Contribución al estudio de los hongos hipogeos de la Comunidad de Madrid. Boletín de la Sociedad Micológica de Madrid 24: 193-201.

Calonge, F.D. \& Pasabán, P.M. 1999. Adiciones al catálogo de hongos de Guipúzcoa y Navarra (Es- 
paña). Registro de cinco especies nuevas. Boletín de la Sociedad Micológica de Madrid 24: 179-185.

Calonge, F.D. \& Sánchez García, M. 1999. Comentarios sobre algunos hongos de los árboles del Real Jardín Botánico de Madrid y tipos de micosis que producen. Boletín de la Sociedad Micológica de Madrid 24: 53-64.

Calonge, F.D., Vasco, F. \& Fernández, A. 1999. Contribución al conocimiento de los hongos hipogeos de Albacete (España). Boletín de la Sociedad Micológica de Madrid 24: 187-191.

Calonge, F.D. \& Vidal, J.M. 1999. Gymnomyces ammophilus Vidal \& Calonge, sp. nov., encontrado en Portugal. Boletín de la Sociedad Micológica de Madrid 24: 65-70.

Moreno-Arroyo, B., Calonge, F.D., Gómez, J \& Pulido, E. 1999. Flora micológica hipogea de Andalucía (España). Boletín de la Sociedad Micológica de Madrid 24: 127-178.

Moreno-Arroyo, B., Gómez, J. \& Calonge, F.D. 1999. Gymnomyces dominguezii sp. nov. from Spain. Mycological Research 103: 215-218.

\section{0}

Calonge, F.D. 2000. A propósito de Tuber macrosporum Vittad. Estudio comparativo con especies próximas. Boletín de la Sociedad Micológica de Madrid 25: 293-294.

Calonge, F.D. 2000. Un caso de envenenamiento leve por ingestión de setas del grupo Clitocybe dealbata. Boletín de la Sociedad Micológica de Madrid 25:325.

Calonge, F.D. 2000. Validation or confirmation of some new taxa recently published. Boletín de la Sociedad Micológica de Madrid 25: 301-302.

Calonge, F.D., Campos, J.C. \& Campos, J.P. 2000. Notas sobre algunos Ascomycetes interesantes recibidos en la Sociedad Micológica de Madrid. Boletín de la Sociedad Micológica de Madrid 25: 15-20.

Calonge, F.D., Fernández, A. \& Vasco, F. 2000. Contribución al conocimiento de los hongos hipogeos de Albacete (España). II. Registro de 10 especies nuevas para la provincia. Boletín de la Sociedad Micológica de Madrid 25: 171-176.

Calonge, F.D., García, F. \& Juste, P. 2000. Adiciones corológicas al catálogo de hongos hipogeos de España. Boletín de la Sociedad Micológica de Madrid 25: 297-299.

Calonge, F.D., García, A., Sanz, M. \& Bastardo, J. 2000. Acutocapillitium filiforme sp. nov. (Lycoperdaceae) della Spagna. Bolletino del Gruppo Micologico G. Brsadola, Nuova Serie 43: 51-57.

Calonge, F.D., Marcos, B., Hernández, E. \& Calabrese, G.M. 2000. Hongos recolectados en encinares (Quercus ilex subsp. ballota), melojares (Q. pyre- naica) y pinares (Pinus sylvestris) de algunas localidades de Salamanca. Boletín de la Sociedad Micológica de Madrid 25: 5-13.

Calonge, F.D. \& Martín, M.P. 2000. Morphological and molecular data on the taxonomy of Gymnomyces, Martellia and Zelleromyces (Russulales). Mycotaxon 76: 9-15.

Calonge, F.D., Moreno-Arroyo, B. \& Gómez, J. 2000. Aportación al conocimiento de los Gasteromycetes, Basidiomycotina de Bolivia (América del Sur). Geastrum ovalisporum sp. nov. Boletín de la Sociedad Micológica de Madrid 25: 271-276.

Calonge, F.D. \& Palacios, D. 2000. Novedades de Gasteromycetes para Navarra. Boletín de la Sociedad Micológica de Madrid 25: 307-308.

Calonge, F.D., Pasabán, P.M. \& Miguel, A. de. 2000. Contribution to the knowledge of the hypogeous fungi of Navarre and surrounding provinces (Northern Spain). Bulletin de la FAMM, N. S. 18: 47-52.

Calonge, F.D. \& Redeuilh, G. 2000. Boletus poikilochromus Pöder, Cetto \& Zuccherelli, una especie mediterránea hallada por primera vez en España. Boletín de la Sociedad Micológica de Madrid 25: 277-279.

Calonge, F.D., Thais Jacobo, M. \& Novoa, D. 2000. Mutinus elegans (Mont.) E. Fischer, un hongo en proceso de expansión en España. Boletín de la Sociedad Micológica de Madrid 25: 287-288.

Calonge, F.D. \& Vidal, J.M. 2000. Contribución al catálogo de los hongos hipogeos de Portugal. Boletín de la Sociedad Micológica de Madrid 25: 251 263.

Calonge, F.D., Vidal, J.M. \& Demoulin, V. 2000. Lycoperdon umbrinoides Dissing \& Lange (Gasteromycetes), a tropical fungus present in Europe. Boletín de la Sociedad Micológica de Madrid 25: 55-58.

Calonge, F.D. \& Zamora, J.C. 2000. Bovista cunninghamii Kreisel (Gasteromycetes), una especie rara en el mundo. Boletín de la Sociedad Micológica de Madrid 25: 295-296.

Martín, M.P. \& Calonge, F.D. 2000. Rhizopogon aromaticus (Boletales, Basidiomycota) a new species found in Spain. Mycotaxon 75: 425-429.

Parent, G.H., Thoen, D. \& Calonge, F.D. 2000. Nouvelles données sur la répartition de Clathrus archeri, en particulier dans l'ouest et le sud-ouest de l'Europe. Bulletin de la Société Mycologique de France 116: 241-266.

\section{1}

Calonge, F.D. \& Vidal, J.M. 2001. Macowanites vinaceodorus sp. nov., (Russulales) a new gasteroid fungus from coastal dunes of Spain. Mycotaxon 79: 1-6. 
Martín, M.P. \& Calonge, F.D. 2001. Rhizopogon buenoi (Boletales, Basidiomycota) a new species from Spain. Mycotaxon 79: 101-105.

\section{2}

Calonge, F.D., García, F. \& Juste, P. 2002. Nuovi dati sui funghi ipogei della Spagna. IX. Pachyphloeus macrosporus sp. nov. Bolletino del Gruppo Micologico G. Brsadola, Nuova Serie 45(2): 51-61.

Calonge, F.D. \& Kreisel, H. 2002. Phallus minusculus sp. nov. from Tropical Africa. Feddes Repertorium 113: 600-602.

Calonge, F.D. \& Mata, M. 2002. Plectania carranzae sp. nov. (Ascomycotina) from Costa Rica. Mycotaxon 81: 237-241.

Calonge, F.D. \& Pérez-de-Gregorio, M.A. 2002. Russula fragrantissima Romagnesi, una especie rara en España. Boletín de la Sociedad Micológica de Madrid 26: 233-234.

Vidal, J.M., Calonge, F.D. \& Martín, M.P. 2002. Macowanites ammophilus (Russulales). A new combination based on new evidence. Revista Catalana de Micologia 24: 69-74.

\section{3}

Calonge, F.D. 2003. In memoriam. Álvaro Zugaza Bilbao (1911-2002). Boletín de la Sociedad Micológica de Madrid 27: 3-5.

Calonge, F.D. 2003. In memoriam. Dr. R.W.G. Dennis (1910-2003). Boletín de la Sociedad Micológica de Madrid 27: 325.

Calonge, F.D. 2003. Un caso de envenenamiento por ingestión de Amanita boudieri. Boletín de la Sociedad Micológica de Madrid 27: 315-317.

Calonge, F.D. 2003. Setas de Madrid. 2. ${ }^{a}$ ed. Consejería de Medio Ambiente. Comunidad de Madrid. Madrid.

Calonge, F.D., Campos, J.C., Prieto, F. \& González, A. 2003. Notas sobre algunos Ascomycetes interesantes recibidos en la Sociedad Micológica de Madrid. II. Scutellinia setosa, Trichophaea paraphysincrustata y Helvella queletii forma alba. Boletín de la Sociedad Micológica de Madrid 27: 309-313.

Calonge, F.D. \& Carranza, J. 2003. Tulostoma matae sp. nov. (Gasteromycetes), found in Costa Rica. Boletin de la Sociedad Micológica de Madrid 27:37- 42.

Calonge, F.D., García, A., Sanz, M. \& Bastardo, J. 2003. Buglossoporus quercinus (Basidiomycotina, Coriolaceae) nueva cita para España. Boletín de la Sociedad Micológica de Madrid 27: 33-35.

Calonge, F.D., García, A., Sanz, M. \& Bastardo, J. 2003. Plectania zugazae (Ascomycotina), especie nueva para la ciencia. Boletín de la Sociedad Micológica de Madrid 27: 17-20.

Calonge, F.D., García, A., Sanz, M. \& Bastardo, J.
2003. Some interesting fungi found in Spain, with special reference to the province of Valladolid. Micologia Italiana 32(2): 45-52.

Calonge, F.D., Iturriaga, T., Mata, M. \& Carranza, J. 2003. Contribución al estudio de los Pezizales (Ascomycotina) de Costa Rica. Boletín de la Sociedad Micológica de Madrid 27: 21-32.

Calonge, F.D. \& Martín, M.P. 2003. Zelleromyces hispanicus, the gasteroid phase of Lactarius aurantiacus. Boletín de la Sociedad Micológica de Madrid 27: 231-236.

Calonge, F.D., Mata, M. \& Carranza, J. 2003. Calvatia sporocristata sp. nov. (Gasteromycetes) from Costa Rica. Revista de Biología Tropical 51: 79-84.

Calonge, F.D. \& Menezes de Sequeira, M. 2003. Contribución al catálogo de los hongos de Madeira (Portugal). Boletín de la Sociedad Micológica de Madrid 27: 277-308.

Calonge, F.D. \& Zamora, J.C. 2003. Geastrum arenarium, encontrado en España y nuevo para Europa. Boletín de la Sociedad Micológica de Madrid 27: 59-61.

\section{4}

Calonge, F.D. 2004. Apuntes para la futura lista roja de hongos españoles. Boletín de la Sociedad Micológica de Madrid 28: 391-395.

Calonge, F.D. 2004. Biodiversidad fúngica y aprovechamiento sostenible de las setas. Anais da Associaçao Micologica "A Pantorra" 4: 45-52.

Calonge, F.D., Guzmán, G. \& Ramírez-Guillén, F. 2004. Observaciones sobre los Gasteromycetes de México depositados en los herbarios XAL y XALU. Boletín de la Sociedad Micológica de Madrid 28: 337-371.

Calonge, F.D., Kreisel, H. \& Guzmán, G. 2004. Bovista sclerocystis, a new species from Mexico. $M y$ cologia 96: 1152-1154.

Calonge, F.D. \& Mata, M. 2004. A new species of Geastrum from Costa Rica and México. Boletín de la Sociedad Micológica de Madrid 28: 331-335.

Medel, R. \& Calonge, F.D. 2004. Aportación al conocimiento de los Discomycetes de México, con especial referencia al género Helvella. Boletín de la Sociedad Micológica de Madrid 28: 151-159.

\section{5}

Baseia, I.G. \& Calonge, F.D. 2005. Aseroë floriformis, a new phalloid with a sunflower-shaped receptacle. Mycotaxon 92: 169-172.

Calonge, F.D. 2005. Funghi con proprietà medicinali. In: Il mondo dei funghi. Ed. del Prado.

Calonge, F.D. 2005. A tentative key to identify the species of Phallus. Boletín de la Sociedad Micológica de Madrid 29: 9-18. 
Calonge, F.D. 2005. Confistulina hepatica, el nombre correcto de la fase mitospórica de Fistulina hepatica. Boletín de la Sociedad Micológica de Madrid 29: 201.

Calonge, F.D. 2005. Tras las setas. Revista de los Paradores Nacionales de Turismo 14: 41-45.

Calonge, F.D. \& Gómez-Ferreras, C. 2005. Helvella corium (Ascomycota), una especie casi desconocida en España. Boletín de la Sociedad Micológica de Madrid 29: 101-104.

Calonge, F.D., Kreisel, H. \& Mata, M. 2005. Phallus atrovolvatus, a new species from Costa Rica. Boletín de la Sociedad Micológica de Madrid 29: 5-8.

Calonge, F.D. \& Mata, M. 2005. Crucibulum laeve var. magnum var. nov. y Arcangeliella scissilis, encontrados en Costa Rica. Boletín de la Sociedad Micológica de Madrid 29: 43-48.

Calonge, F.D., Mata, M. \& Carranza, J. 2005. Contribución al catálogo de los Gasteromycetes (Basidiomycotina, Fungi) de Costa Rica. Anales del Jardín Botánico de Madrid 62: 23-45.

Calonge, F.D. \& Pasabán, P.M. 2005. Macowanites candidus (Russulales), nuevo para el catálogo micológico español. Boletín de la Sociedad Micológica de Madrid 29: 87-90.

Calonge, F.D. \& Vidal, J.M. 2005. Gastrolactarius denudatus (Basidiomycotina, Russulales), a new species from México. Revista Mexicana de Micología 21:59-62.

[Diego] Calonge, F. de. 2005. Culvatia booniana. Boletín de la Sociedad Micológica Extremeña 16: 17-18.

Douanla-Meli, C., Langer, E. \& Calonge, F.D. 2005. Geastrum pleosporus, sp. nov., a new species of Geastraceae identified by morphological and molecular phylogenetic data. Mycological Progress 4: 239-250.

García, F. \& Calonge, F.D. 2005. Encuesta sobre el consumo de Tricholoma equestre y otras setas, en la Sociedad Micológica de Madrid, durante la primavera de 2002. Boletín de la Sociedad Micológica de Madrid 29: 91-100.

Kreisel, H., Calonge, F.D. \& Pasabán, P.M. 2005. Ptychogaster rubescens, el anamorfo de Oligoporus floriformis, presente en España. Boletín de la Sociedad Micológica de Madrid 29: 27-30.

Martín, M.P., Calonge, F.D. \& Marcos, B. 2005. The limits between Lysurus cruciatus and L. cruciatus var. nanus. A comparative DNA sequential study. Boletín de la Sociedad Micológica de Madrid 29: 31-36.

Moreno-Arroyo, B., Gómez, J. \& Calonge, F.D. 2005. Modicella malleola (Mortierellaceae), nuevo registro para España. Boletín de la Sociedad Micológica de Madrid 29: 197-198.

\section{6}

Baseia, I.G. \& Calonge, F.D. 2006. Geastrum hirsutum: a new earthstar fungus with a hairy exoperidium. Mycotaxon 95: 301-304.

Baseia, I.G., Cortez, V.G. \& Calonge, F.D. 2006. Rick's species revision: Mitremyces zanchianus versus Calostoma zanchianum. Mycotaxon 95: 113-116.

Baseia, I.G., Maia, L.C. \& Calonge, F.D. 2006. Notes on Phallales in the neotropics. Boletín de la Sociedad Micológica de Madrid 30: 87-93.

Calonge, F.D. 2006. In memoriam. Prof. Emilio Fernández-Galiano Fernández. Anales del Jardín Botánico de Madrid 63: 253-256.

Calonge, F.D., Campos, J.C. \& Jerez, M. 2006. Sowerbyella radiculata (Ascomycota, Pezizales), una especie rara en España, localizada en CastillaLa Mancha. Boletín de la Sociedad Micológica de Madrid 30: 13-16.

Calonge, F.D., González, A. \& Prieto-García, F. 2006. Aproximación al catálogo de Gasteromycetes s.l. de Castilla-La Mancha. Boletín de la Sociedad Micológica de Madrid 30: 99-110.

Calonge, F.D., González, A., Prieto-García, E. \& Hernández-Crespo, J.C. 2006. Hongos de CastillaLa Mancha I: El género Scutellinia (Ascomycota, Pezizales). Boletín de la Sociedad Micológica de Madrid 30: 43-47.

Calonge, F.D. \& Gonçalves Silva, J.J. 2006. Lysurus mokusin, Phallales, Basidiomycota, especie nueva para la isla de Madeira (Portugal). Boletín de la Sociedad Micológica de Madrid 30: 95-97.

Calonge, F.D. \& Mata, M. 2006. Adiciones y correcciones al catálogo de Gasteromycetes de Costa Rica. Boletín de la Sociedad Micológica de Madrid 30: 111-119.

Calonge, F.D., Mata, M. \& Umaña, L. 2006. Adiciones y correcciones al catálogo de Ascomycota (Fungi) de Costa Rica, con especial referencia al género Scutellinia. Boletín de la Sociedad Micológica de Madrid 30: 25-34.

Calonge, F.D., Mata, M. \& Umaña, L. 2006. El género Phillipsia (Ascomycota) en Costa Rica, con una clave para identificar las especies. Boletín de la Sociedad Micológica de Madrid 30: 35-42.

Calonge, F.D., Vidal, J.M. \& Campos, J.C. 2006. Stephensia bombycina (Ascomycota, Pezizales) una especie rara en España. Boletín de la Sociedad Micológica de Madrid 30: 9-11.

Martín, M.P. \& Calonge, F.D. 2006. Rhizopogon buenoi, segunda cita mundial, encontrado en CastillaLa Mancha (España). Boletín de la Sociedad Micológica de Madrid 30: 319-321.

Medel, R., Calonge, F.D. \& Guzmán, G. 2006. Nuevos registros de Pezizales (Ascomycotina) de Veracruz. Revista Mexicana de Micología 23: 83-86. 
2007

Calonge, F.D., González, A. \& Prieto-García, F. 2007. Neournula pouchetii (Ascomycota), una especie rara en España, encontrada en Castilla-La Mancha. Boletín de la Sociedad Micológica de Madrid 31:3-5.

Calonge, F.D., Guzmán, G., Ramírez-Guillén, F. \& Gándara, E. 2007. Adiciones al catálogo de Gasteromycetes de México, con referencia especial a los géneros Blumenavia y Tulostoma. Boletín de la Sociedad Micológica de Madrid 31: 151-155.

Calonge, F.D., Llimona, X. \& Martín, M.P. 2007. Nuevos datos sobre el género Tulostoma (Gasteromycetes) en España. Revista Catalana de Micologia 29: 11-16.

Calonge, F.D., Mata, M. \& Umaña, L. 2007. Macrocybe titans, un hongo espectacular presente en Costa Rica, América Central. Bulletim de la FAMM, N.S. 32: 21-24.

Calonge, F.D. \& Menezes de Sequeira, M. 2007. Adiciones y correcciones al catálogo micológico de Madeira (Portugal). Boletín de la Sociedad Micológica de Madrid 31: 231-238.

Cortez, V.G., Calonge, F.D. \& Baseia, I. 2007. Rick's species revision 2: Lycoperdon bejaminii recombined in Morganella. Mycotaxon 102: 425-429.

Das, K., Singh, S.K. \& Calonge, F.D. 2007. Gasteromycetes of Western Ghats, India: I. A new form of Phallus indusiatus. Boletín de la Sociedad Micológica de Madrid 31: 135-138.

Leite, A.G., Calonge, F.D. \& Baseia, I.G. 2007. Additional studies on Geastrum from northeastern Brazil. Mycotaxon 101: 103-111.

Silva, B.D.B., Calonge, F.D. \& Baseia, I.G. 2007. Studies on Tulostoma (Gasteromycetes) in the neotropics. Some Brazilian species. Mycotaxon 101: 47-54.

Zamora, J.C. \& Calonge, F.D. 2007. Geastrum parvistriatum, una especie nueva encontrada en España. Boletín de la Sociedad Micológica de Madrid 31: 139-149.

\section{8}

Baseia, I.G. \& Calonge, F.D. 2008. Calvatia sculpata, a striking puffball occurring on Brazilian san dunes. Mycotaxon 106: 269-272.

Calonge, F.D. \& Barrio de la Parte, L. 2008. Pachyphloeus macrosporus (Ascomycota), encontrado en el Norte de España y segunda cita mundial. Boletín de la Sociedad Micológica de Madrid 32: 11-12.

Calonge, F.D., Iturriaga, T., Mata, M. \& Carranza, J. 2008. Peziza disparispora (Ascomycota), a new species from Costa Rica. Mycologia Montenegrina 10: 23-30 [2007].

Calonge, F.D., Menezes de Sequeira, M., Aguiar, G. \& Rocha, E. 2008. Adiciones al catálogo micológico de Madeira (Portugal). II. Registro de 23 especies nuevas. Boletín de la Sociedad Micológica de Madrid 32: 249-259.

Calonge, F.D., Menezes de Sequeira, M., Freitas, T., Rocha, E. \& Franquinho, L. 2008. Phallus maderensis sp. nov., found in Madeira, Portugal. Boletín de la Sociedad Micológica de Madrid 32: 101104.

Calonge, F.D., Moreno-Arroyo, B. \& Gómez, J. 2008. Estudios sobre hongos tropicales. Algunas especies colectadas en Ecuador y Guatemala. Boletin de la Sociedad Micológica de Madrid 32: 97-100.

Calonge, F.D., Prieto-García, F. \& González, A. 2008 Lenzites warnieri (Polyporaceae), segunda cita peninsular, encontrado en Castilla-La Mancha. Boletín de la Sociedad Micológica de Madrid 32: 81-84.

Calonge, F.D. \& Syvokon, E.V. 2008. Contribution to the knowledge of the Gasteromycetes, Basidiomycota, from Ukraine. Boletín de la Sociedad Micológica de Madrid 32: 91-95.

[Diego] Calonge, F. de. 2008. Pinceladas sobre la micología en España. Boletín de la Sociedad de Amigos del Real Jardín Botánico 9: 1-2.

Fazolino, E.P., Calonge, F.D. \& Baseia, I.G. 2008. Geastrum entomophilum, a new earthstar with an unusual spore dispersal strategy. Mycotaxon 104: 449-453. 